\title{
Simultaneous degradation of non-emissive and emissive dyes on visible light illuminated $\mathrm{TiO}_{2}$ surface
}

\author{
Debabrata Chatterjee $^{\mathrm{a}, *}$, Shimanti Dasgupta ${ }^{\mathrm{a}}$, Rita S. Dhodapkar ${ }^{\mathrm{b}}$, Nageswar N. Rao ${ }^{\mathrm{b}}$ \\ ${ }^{a}$ Chemistry Section, Central Mechanical Engineering Research Institute, Durgapur 713209, India \\ ${ }^{\mathrm{b}}$ Wastewater Technology Division, National Environmental Engineering Research Institute, Nagpur 400020, India \\ Received 30 April 2006; received in revised form 13 July 2006; accepted 16 July 2006 \\ Available online 1 September 2006
}

\begin{abstract}
Simultaneous photocatalytic degradation of non-emissive dye, acid blue1 (AB1) and emissive dyes (eosinY or thionine) in air-equilibrated aqueous suspension of $\mathrm{TiO}_{2}$ semiconductor photocatalyst has been achieved at ambient conditions. Under visible light irradiation AB1 undergoes appreciable degradation in presence of a sensitizing dye (eosinY or thionine). However, concomitant decoloration of eosinY (EY) or thionine (Th) due to self-sensitized degradation was also noticed. Mechanistic proposals for the photocatalytic degradation of AB1 using a $150 \mathrm{~W}$ Xe lamp with a UV-filter $(\lambda>420 \mathrm{~nm})$ as well as without a UV-filter, are discussed. Formation of reactive ${ }^{\circ} \mathrm{O}_{2}{ }^{-} /{ }^{\bullet} \mathrm{HO}_{2}$ radicals is proposed to be responsible for the degradation of the selected dyes, AB1, EY and Th.
\end{abstract}

(C) 2006 Elsevier B.V. All rights reserved.

Keywords: Photodegradation; Dye sensitization; Acid blue1; $\mathrm{TiO}_{2}$ semiconductor; Visible light

\section{Introduction}

Design and development of photocatalysts for degradation of contaminants in wastewater over visible light irradiation is a demanding area of research for harvesting maximum part of the solar energy [1]. The vital snag of $\mathrm{TiO}_{2}$ semiconductor is that it absorbs a small portion of solar spectrum in the UV region (band gap energy of $\mathrm{TiO}_{2}$ is $3.2 \mathrm{eV}$ ). In order to exploit the more abundant source of light energy coming from the sun certain changes will have to be incorporated within the semiconductors system so as to make it suitable for receiving and utilizing solar energy with good efficiency. Dye sensitization [2] seems to be a viable alternative method for contending with this issue.

Very recently we explored the application of dye sensitization technique to degradation of colorless organics, which absorb light in the UV region, using visible light illuminated dye modified $\mathrm{TiO}_{2}$ photocatalyst [1]. In the present work viability of dye sensitized photocatalytic degradation of a non-emissive dye, acid blue 1 (AB1) over visible light illuminated $\mathrm{TiO}_{2}$ pho-

\footnotetext{
* Corresponding author.

E-mail address: dchat57@ hotmail.com (D. Chatterjee).
}

tocatalyst is examined. A number of reports on the UV-light assisted photocatalytic degradation of acid blue dyes typical of anthraquinonic (AB40 [3], AB80 [4], AB25 [5]) and indigoid $(\mathrm{AB} 74[6,7])$ groups are available in the literature. However, a report on the photocatalytic degradation of AB1 (Colour Index No: 42045) which belongs to a very important class of dyes (triarylmethane) that are mainly used in detergents, soaps, inks, and leather is conspicuous by its absence, although degradation of a structurally similar acid blue dye (AB9) using Fenton's reagent has been reported in the literature [8]. We report herein eosinY (EY) or thionine (Th) sensitized degradation of $\mathrm{AB} 1$ in aqueous suspensions of $\mathrm{TiO}_{2}$ under visible light irradiated photolysis. The novelty of the present work lies in its simplicity by which a non-sensitizing dye in an effluent can be degraded by just mixing with another effluent containing sensitizing dye and subjecting the mixture of effluents to visible light photolysis over $\mathrm{TiO}_{2}$ semiconductor photocatalysts.

\section{Experimental}

\subsection{Materials and methods}

All the chemicals used in the present study were of A.R. grade and doubly distilled water was used throughout the exper- 
iment. Spectral (UV-vis) measurements were carried out with a Perkin-Elmer (Model Lambda 35) spectrophotometer. Fluorescence measurements were performed using a Hitachi F-4010 spectrofluorometer in the Chemistry Department, Burdwan University.

\subsection{Photodegradation of pollutants}

In a typical photocatalysis experiment $50 \mathrm{mg}$ of $\mathrm{TiO}_{2}$ semiconductor photocatalyst was added into an aqueous solution $(50 \mathrm{ml})$ that contained sensitizer dye $(\mathrm{D}=\mathrm{EY}, \mathrm{Th})$ and $\mathrm{AB} 1$ (Fig. 1). The resultant mixture was taken in a flat-surfaced glass reactor. The pre-aerated reaction mixture was illuminated with a $150 \mathrm{~W}$ xenon lamp (Oriel Instruments) under continuous magnetic stirring. A filter solution containing sodium nitrite, copper sulfate and ammonium hydroxide was used as a UV-filter [9] to eliminate light $<420 \mathrm{~nm}$. Oxygen was bubbled into the photoreactor. The $\mathrm{pH}$ of reacting system was controlled at 5.0 by using $0.1 \mathrm{M} \mathrm{NaOH}$. After chosen interval of irradiation time aliquots of reaction mixture were withdrawn and filtered by using a membrane filter $(0.1 \mu \mathrm{m})$ and the depletion of dye was estimated spectrophotometrically at the absorption maximum $\left(\lambda_{\max }\right)$ of each dye. The evolved gas was flushed with oxygen through $\mathrm{BaCl}_{2}$ solution and production of $\mathrm{CO}_{2}$ was verified with the formation of $\mathrm{BaCO}_{3}$.

\section{Results and discussion}

Absorption and emission spectra of Th and EY obtained in aqueous solutions were found to be in good agreement with the spectral data $\left(\lambda_{\mathrm{abs}}=518 \mathrm{~nm}, \lambda_{\text {emiss }}=546 \mathrm{~nm}\right.$ for EY; $\lambda_{\mathrm{abs}}=600 \mathrm{~nm}, \lambda_{\text {emiss }}=622 \mathrm{~nm}$ for Th) reported earlier [10]. The photoexcited state redox properties of EY and Th are different

Table 1

Adsorption of dyes on the surface of $\mathrm{TiO}_{2}$ in dark

\begin{tabular}{lllc}
\hline Dye & \multicolumn{2}{l}{$\%$ adsorption } \\
\cline { 2 - 4 } & AB1 & EY & Th \\
\hline AB1 & 2.62 & - & - \\
EY & - & 4.54 & - \\
Th & - & - & 12.1 \\
AB1 + EY & 1.57 & 3.48 & - \\
AB1 + Th & 0.5 & - & 9.7 \\
\hline
\end{tabular}

Table 2

Results of decoloration of $\mathrm{AB} 1$ in the presence of eosin $\mathrm{Y}^{\mathrm{a}}$<smiles>Nc1ccc2nc3ccc(=[NH2+])cc-3sc2c1</smiles><smiles>O=C([O-])c1ccccc1-c1c2cc(Br)c(=O)c(Br)c-2oc2c(Br)c(O)c(Br)cc12</smiles>

Eosin-Y<smiles>CCN(CC)c1ccc(C(=C2C=CC(=[N+](CC)CC)C=C2)c2ccc([N+](=O)[O-])cc2)cc1</smiles>

Acid Blue1

Fig. 1. Structures of the various dye species.

with regard to their excited state electron transfer processes. Thionine at the excited state is known to accept one electron and converts into semithionine [11], whereas EY gives up one electron upon excitation with visible light [12].

Results of adsorption of AB1 and EY studied under various experimental conditions are summarized in Table 1. Experiments with $\mathrm{TiO}_{2}$ in dark reveal that in an aqueous mixture of $\mathrm{AB} 1$ and $\mathrm{EY}$ (present at equal concentration of $25 \mathrm{ppm}$ ), EY was adsorbed more on to the surface of $\mathrm{TiO}_{2}$ photocatalyst than AB1 (Table 1). No change in the spectral pattern observed after $5 \mathrm{~h}$ of stirring the aqueous solution of $\mathrm{AB} 1$ and $\mathrm{EY}$ in dark indicates that $\mathrm{AB} 1$ and $\mathrm{EY}$ did not interact with each other in dark. Prolonged illumination $(5 \mathrm{~h})$ of the same reaction mixture did not cause any appreciable loss of spectral features of either of the dyes in the reaction mixture. This suggests that eosinY, a typical electron donor in the excited state [12] cannot oxidize $\mathrm{AB} 1$ in the presence of light. No decolorization of AB1 was observed when an its aqueous solution was photolyzed separately under visible light illumination $(>420 \mathrm{~nm})$, though EY underwent self-sensitized degradation under similar conditions (Table 2). However, photolysis of the mixture of $\mathrm{AB} 1$ and $\mathrm{EY}$ resulted in an appreciable degradation of both EY

\begin{tabular}{|c|c|c|c|c|c|c|c|c|}
\hline \multirow[t]{4}{*}{ Catalyst } & \multicolumn{8}{|c|}{$\%$ decoloration ${ }^{\mathrm{b}}$} \\
\hline & \multicolumn{4}{|c|}{ Visible light (>420 nm) } & \multicolumn{4}{|c|}{ UV-vis (without UV-filter) } \\
\hline & \multirow[t]{2}{*}{$\mathrm{AB} 1$} & \multirow[t]{2}{*}{ EY } & \multicolumn{2}{|c|}{ Mixture of $\mathrm{AB} 1$ and $\mathrm{EY}$} & \multirow[t]{2}{*}{$\mathrm{AB} 1$} & \multirow[t]{2}{*}{$\mathrm{EY}$} & \multicolumn{2}{|c|}{ Mixture of $\mathrm{AB} 1$ and $\mathrm{EY}$} \\
\hline & & & $\mathrm{AB} 1$ & EY & & & $\mathrm{AB} 1$ & EY \\
\hline $\mathrm{No} \mathrm{TiO}_{2}$ & Nil & Nil & Nil & Nil & Nil & Nil & Nil & Nil \\
\hline $\mathrm{TiO}_{2}$ & Nil & 70.1 & 56.7 & 65.7 & 33.1 & 80.5 & 63.0 & 71.3 \\
\hline
\end{tabular}

\footnotetext{
a $5 \mathrm{~h}$ photolysis, concentration of dyes in all cases was kept at $25 \mathrm{ppm}$.
}

b Based on initial dye concentration (25 ppm). 


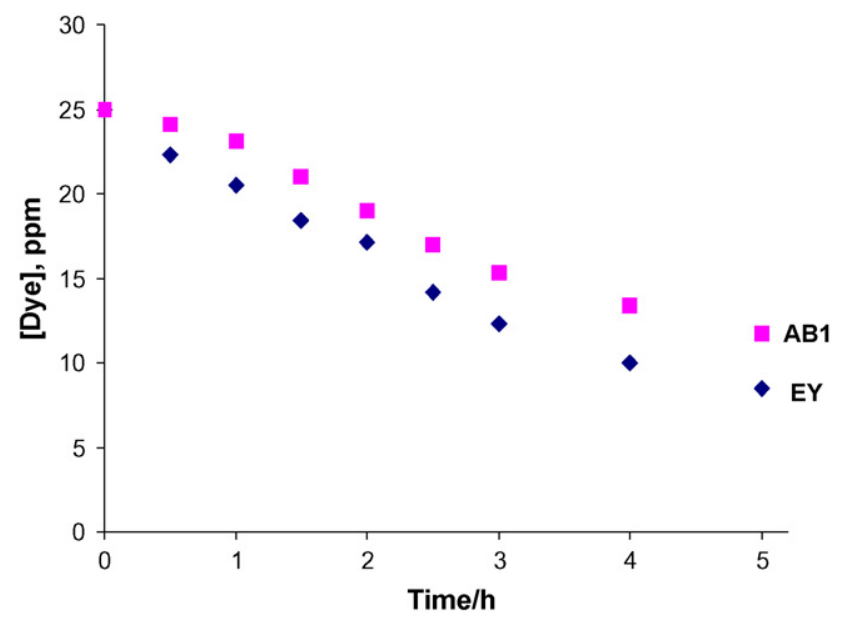

Fig. 2. Decrease in dye concentration with time during visible light irradiation $(\lambda>420 \mathrm{~nm})$ of a mixture $(50 \mathrm{ml})$ of two dyes AB1 and EY present in equal concentration $(25 \mathrm{ppm})$.

and AB1 (Table 2). Decrease in the dye concentration with time is shown in Fig. 2. Formation of carbon dioxide as evidenced by the $\mathrm{BaCO}_{3}$ test undeniably substantiated the degradation of dyes in the reaction mixture under specified conditions. In the absence of oxygen photodegradation of the selected dyes was found to be negligible. Based on the present experimental results the following working mechanism (Scheme 1) is proposed for the photocatalytic degradation of $\mathrm{AB} 1$ and $\mathrm{EY}$, both under visible light irradiation.

The EY adsorbed at the surface of $\mathrm{TiO}_{2}$ gets excited and injects one electron to the conduction band [12] as outlined in Eqs. (1) and (2) and dioxygen takes up the electron to produce ${ }^{-} \mathrm{O}_{2}{ }^{-}$radical $[13,14]$, which subsequently reacts with $\mathrm{EY}$ as well $\mathrm{AB} 1$ to effect their degradation. The results of the present studies reveal that $\mathrm{EY}$ is kinetically more vulnerable towards $\bullet \mathrm{O}_{2}-/{ }^{-} \mathrm{HO}_{2}$ radicals attack than $\mathrm{AB} 1$, as the extent of degradation of $\mathrm{EY}$ is always greater than that of $\mathrm{AB} 1$ irrespective of the nature of irradiation (Table 2).

When an aqueous solution containing $\mathrm{AB} 1$ and $\mathrm{Th}$ at an equal concentration ( $25 \mathrm{ppm}$ ) was subjected to prolonged photolysis, decolorization of the reaction mixture was observed (Table 3). This degradation of $\mathrm{AB} 1$ in the absence of $\mathrm{TiO}_{2}$ is consistent with the excited state redox properties of the thionine, which accepts electron at the excited state. However, as observed experimentally, eosinY usually gives up electron at the excited state and so cannot oxidize $\mathrm{AB} 1$ to $\mathrm{AB} 1^{+\bullet}$ upon irradiation in the absence of $\mathrm{TiO}_{2}$. This is consistent with the fact that thionine, upon photoexcitation, oxidizes $\mathrm{AB} 1$ to $\mathrm{AB} 1^{+\bullet}$, and in the process it is converted into a colorless species, semithionine [11]. The results of visible light assisted degradation of $\mathrm{AB} 1$ and $\mathrm{Th}$ in the presence of $\mathrm{TiO}_{2}$ photocatalyst are summarized in Table 3. Time course of decolorization of Th and AB1 together is shown in Fig. 3. Considering photo-excited Th is a typical electron acceptor, degradation $\mathrm{AB} 1$ in the pres-

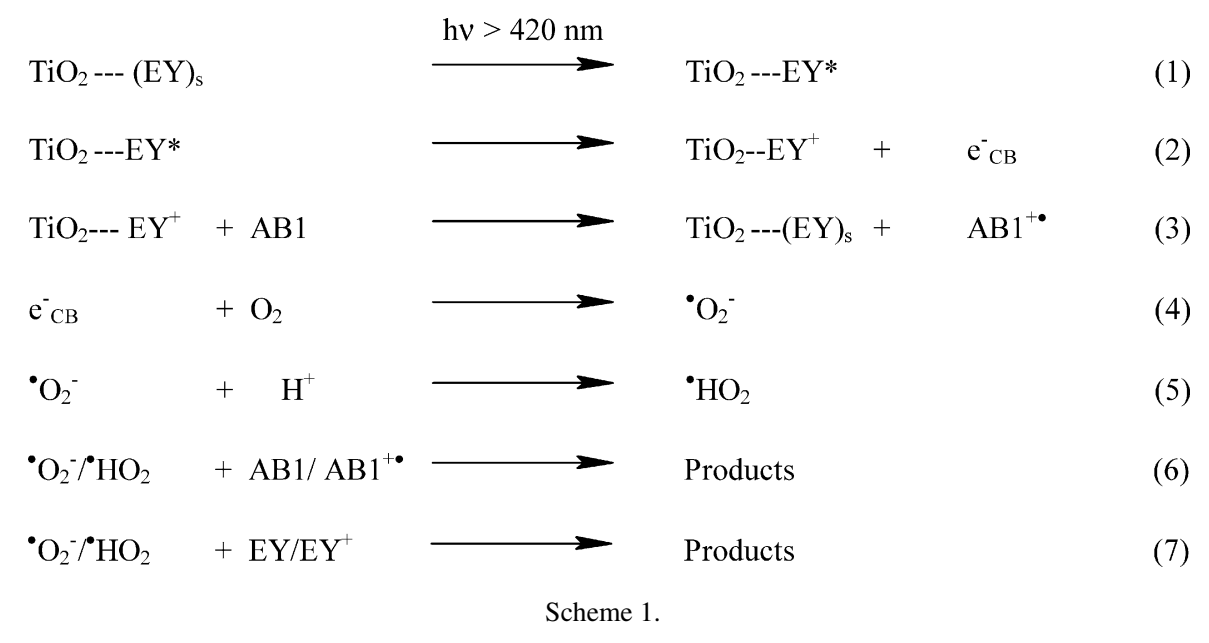

Table 3

Results of decoloration of $\mathrm{AB} 1$ in the presence of thionine $\mathrm{a}^{\mathrm{a}}$

\begin{tabular}{|c|c|c|c|c|c|c|c|c|}
\hline \multirow[t]{4}{*}{ Catalyst } & \multicolumn{8}{|c|}{$\%$ decoloration ${ }^{\mathrm{b}}$} \\
\hline & \multicolumn{4}{|c|}{ Visible light (>420 nm) } & \multicolumn{4}{|c|}{ UV-vis (without UV-filter) } \\
\hline & \multirow[t]{2}{*}{$\mathrm{AB} 1$} & \multirow[t]{2}{*}{ Th } & \multicolumn{2}{|c|}{ Mixture of $\mathrm{AB} 1$ and $\mathrm{Th}$} & \multirow[t]{2}{*}{$\mathrm{AB} 1$} & \multirow[t]{2}{*}{ Th } & \multicolumn{2}{|c|}{ Mixture of $\mathrm{AB} 1$ and $\mathrm{Th}$} \\
\hline & & & $\mathrm{AB} 1$ & Th & & & $\mathrm{AB} 1$ & Th \\
\hline No $\mathrm{TiO}_{2}$ & Nil & Nil & 12.5 & 17.3 & Nil & Nil & 14 & 19 \\
\hline $\mathrm{TiO}_{2}$ & Nil & 63 & 41 & 65.2 & 33.1 & 80 & 47 & 75 \\
\hline
\end{tabular}

\footnotetext{
a $5 \mathrm{~h}$ photolysis, concentration of dyes in all cases was kept at $25 \mathrm{ppm}$.
}

${ }^{\mathrm{b}}$ Based on initial dye concentration (25 ppm). 


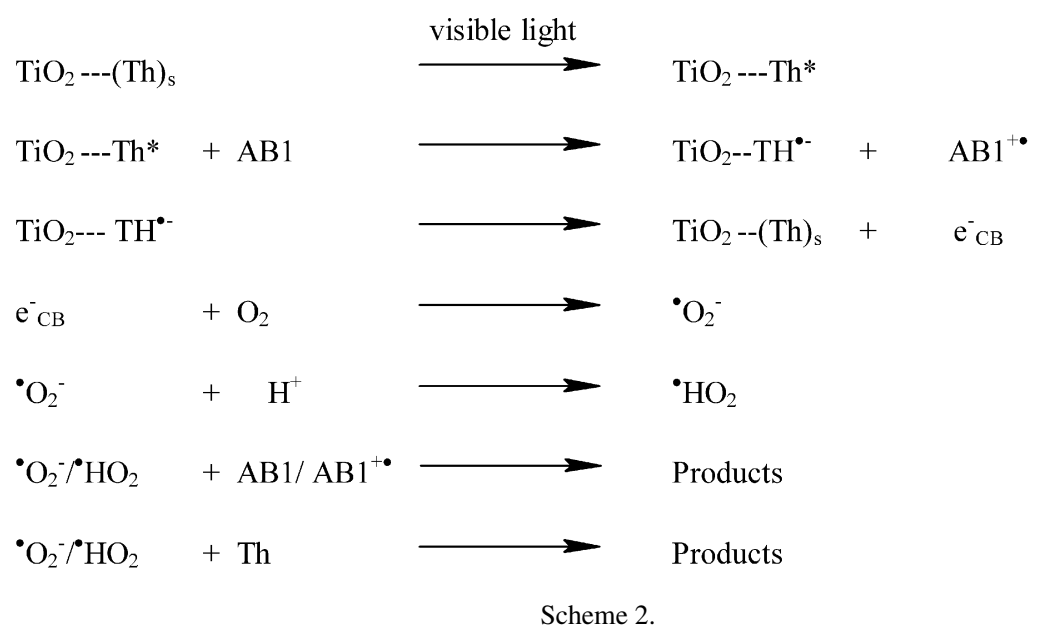

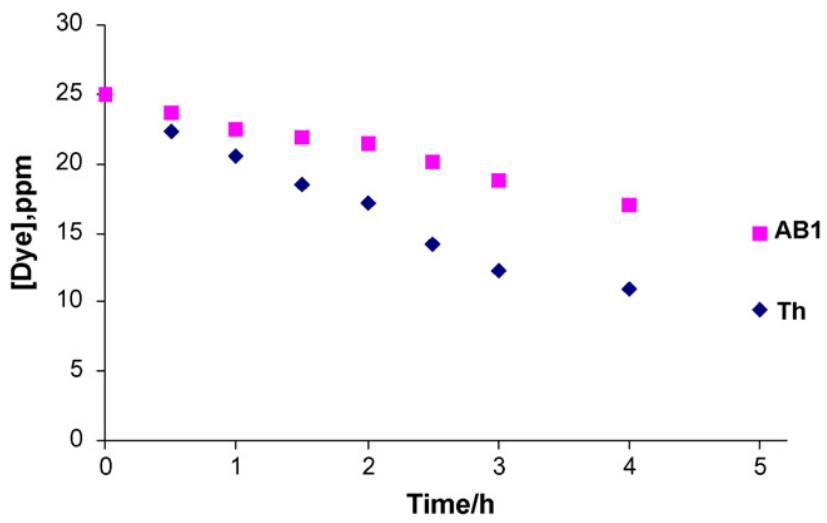

Fig. 3. Decrease in dye concentration with time during visible light irradiation $(\lambda>420 \mathrm{~nm})$ of a mixture $(50 \mathrm{ml})$ of two dyes $\mathrm{AB} 1$ and Th present in equal concentration $(25 \mathrm{ppm})$.

ence Th under visible light irradiated conditions is illustrated in Scheme 2.

As proposed in Scheme 2 illumination of the catalytic system with visible light $(h v)$ results in the excitation of the surface adsorbed thionine (Eq. (8)), which, like the visible light irradiated homogeneous $\mathrm{AB} 1 / \mathrm{Th}$ system, reacts with the reducing AB1 molecule to form semithionine (Eq. (9)). Semithionine then effects charge transition into the conduction band (Eq. (10)). Empty conduction band edge of $\mathrm{TiO}_{2}\left(E_{\mathrm{CB}}=-0.34 \mathrm{~V}\right.$ versus NHE at $\mathrm{pH}$ 5.0) [10] seems to be thermodynamically competent to oxidize one electron reduced surface adsorbed thionine (reduction potential of thionine is $0.064 \mathrm{~V}$ versus NHE) [10].

However, both $\mathrm{AB} 1$ and $\mathrm{Th}$ is reasonably more degraded when irradiated without a UV-filter. This is plausibly explicable by considering that in the case of irradiation without a UV-filter the oxidation involving $\mathrm{e}^{-}{ }_{\mathrm{CB}} / \mathrm{h}^{+}$vB pair created by the UV portion of the light (however small) is also operative in conjunction with dye-sensitized (driven by the visible part of the light) oxidation. Absorption of light $(<420 \mathrm{~nm})$ by the colorless semithionine species in fostering the charge transfer process further cannot be ruled out in the overall photodegradation process.

\section{Conclusion}

The results of the present study obviously demonstrate for the first time that in presence of sensitizing dyes like EY (or Th) photocatalytic degradation of acid blue1 in water is achievable by using a visible light irradiated $\mathrm{TiO}_{2}$ semiconductor particulate system. Even if complete degradation is not achieved for the selected colorants, the present findings are of significance and prospect. It affords an effective method of degrading non-sensitizing dye in an effluent mixed with another effluent that contains sensitizing dye using the sunlight irradiated $\mathrm{TiO}_{2}$ photocatalytic system. Efficiency of such photocatalytic systems in the degradation of AB1 (or such nonemissive dyes) mostly depends on the extent of adsorption and excited state redox potential of sensitizing dye. Studies with real dying and finishing effluents pertinent to this matter are in progress.

\section{Acknowledgments}

Authors are thankful to Dr. G.P. Sinha, Director, CMERI, Durgapur and Dr. S.K. Devotta, Director, NEERI, Nagpur for their encouragement. SD thanks CMERI for internship. This work was carried out under the financial support from CSIR (India) Network Project (CORE-08, Industrial Waste Minimization and Cleanup).

\section{References}

[1] D. Chatterjee, S. Dasgupta, J. Photochem. Photobiol. C. Photochem. Rev. 6 (2005) 186.

[2] K. Kalyanasundaram, M. Gratzel, Coord. Chem. Rev. 77 (1998) 347.

[3] W.J. Tang, H. An, Chemosphere 31 (1995) 4171.

[4] A.B. Prevot, C. Baiocchi, M.C. Brussino, E. Pramauro, P. Savarino, V. Augugliaro, G. Marci, L. Palmisano, Environ. Sci. Technol. 35 (2001) 971.

[5] I. Bouzaida, C. Ferronato, J.M. Chovelon, M.E. Rammah, J.M. Herrmann, J. Photochem. Photobiol. A: Chem. 168 (2004) 23.

[6] C. Galindo, P. Jacques, A. Kalt, Photobiol. A: Chem. 141 (2001) 47.

[7] A. Aleboyeh, Y. Moussa, H. Aleboyeh, Dye Pigm. 66 (2005) 129.

[8] M. Jank, H. Koser, F. Lucking, M. Martienssen, S. Wittchen, Environ. Sci. Technol. 19 (1998) 741. 
[9] P.V. Kamat, in: D.F. Ollis, H. Al-Ekabi (Eds.), Photocatalytic Purification and Treatment of Water and Air, Elsevier Science Publishers BV, Amsterdam, The Netherlands, 1993, p. p455.

[10] M.A. Fox, M.T. Dulay, J. Photochem. Photobiol. A: Chem. 98 (1996) 91.

[11] G.R. Brown, F.R. Darwent, P.D.I. Fletcher, J. Chem. Soc. 107 (1985) 6446.
[12] R. Abe, K. Hara, K. Sayama, K. Domen, H. Arakawa, J. Photochem. Photobiol. A: Chem. 137 (2000) 63.

[13] D. Chatterjee, A. Mahata, J. Photochem. Photobiol. Chem.: A 165 (2004) 19.

[14] H. Gerischer, A. Heller, J. Phys. Chem. 95 (1991) 5261. 